\title{
General relation between Aretakis charge and Newman-Penrose charge
}

\author{
Mirjam Cvetič ${ }^{1}$ and Alejandro Satz ${ }^{2}$ \\ ${ }^{1}$ Department of Physics and Astronomy, University of Pennsylvania, Philadelphia, Pennsylvania 19104, USA \\ and Center for Applied Mathematics and Theoretical Physics, University of Maribor, \\ SI2000 Maribor, Slovenia \\ ${ }^{2}$ Sarah Lawrence College, Bronxville, NY 10708, USA \& Department of Physics and Astronomy, \\ University of Pennsylvania, Philadelphia, Pennsylvania 19104, USA
}

(Received 2 December 2018; published 26 December 2018)

\begin{abstract}
We reexamine the relation between the Aretakis charge of an extremal black hole spacetime and the Newman-Penrose charge of a weakly asymptotically flat spacetime obtained from the original one through radial inversion and conformal mapping. Building on recent work by Godazgar, Godazgar, and Pope, we present an explicit general relation between these quantities showing how the charge densities are mapped. As a nontrivial example we provide the computation of both quantities and their explicit relation for the extremal Kerr spacetime.
\end{abstract}

DOI: 10.1103/PhysRevD.98.124035

\section{INTRODUCTION}

As part of his analyses showing the instability of extreme black hole horizons under perturbations, Aretakis showed that fields propagating on the extreme black hole background have a conserved horizon charge [1-3]. This socalled Aretakis charge has been shown to exist in higher dimensions as well as four [4], and for massless and massive scalars as well as gauge fields [5,6]. Moreover, although the Aretakis charge involves in its definition only the field and its radial derivative at the horizon, it is only the first in an infinite hierarchy of conserved charges involving higher radial derivatives $[3,5]$.

Shortly after the discovery of the Aretakis charges, it was shown [6,7] that for extremal Reissner-Nordström this charges can be mapped, through a conformal symmetry of the metric under radial inversion, to Newman-Penrose charges at future asymptotic null infinity. Higher multipoles of this mapping were computed in [8]. In addition, it has been argued in [9] that the Aretakis charge of any extreme black hole can be put in correspondence, through a similar procedure, with the Newman-Penrose charge of a dual weakly asymptotic spacetime. However, no other explicit examples than the Reissner-Nordström were computed.

The goal of this paper is to extend and refine the conclusions of [9]. First, we make fully explicit the relation between the Aretakis charge of a general extreme

Published by the American Physical Society under the terms of the Creative Commons Attribution 4.0 International license. Further distribution of this work must maintain attribution to the author(s) and the published article's title, journal citation, and DOI. Funded by SCOAP ${ }^{3}$. four-dimensional black hole and the Newman-Penrose charge in an associated weakly asymptotically flat spacetime, which is conformally related to the radial inversion of the original spacetime. Second, we provide a new nontrivial example by exhibiting this relation for the extremal Kerr black hole. A main conclusion of our work is that when the black hole is not spherically symmetric there is no direct mapping between the two charges but rather a more subtle relation between the charge densities.

In the next section, we establish our framework and summarize the main results of [9]. In Sec. III, we present the general relation between the Aretakis and the NewmanPenrose charges, as well as its explicit computation for the extreme Kerr black hole. Section IV contains a summary and discussion of the results.

\section{DUALITY BETWEEN EXTREMAL HORIZON AND ASYMPTOTIC INFINITY}

In this section we summarize the results of [9] concerning the duality between the Aretakis charge of an extremal black hole and the asymptotic Newman-Penrose charge of a dual conformal metric.

Consider a 4-dimensional extremal black hole with a Killing horizon. In the vicinity of the horizon one can introduce Gaussian null coordinates $\left(v, \rho, x^{i}\right)$, with $i=(1,2)$. The horizon corresponds to $\rho=0$, and near it the metric takes the form:

$$
\begin{aligned}
d s^{2}= & L\left(x^{i}\right)^{2}\left[-\rho^{2} F\left(\rho, x^{i}\right) d v^{2}+2 d v d \rho\right] \\
& +\gamma_{i j}\left(\rho, x^{i}\right)\left(d x^{i}-\rho h^{i}\left(\rho, x^{i}\right) d v\right)\left(d x^{j}-\rho h^{j}\left(\rho, x^{i}\right) d v\right),
\end{aligned}
$$


with $F\left(\rho=0, x^{i}\right)=1$. It is assumed that $h^{i}\left(\rho, x^{i}\right)=O(1)$ at the horizon, and that $\gamma_{i j}\left(\rho=0, x^{i}\right)$ is a topologically spherical metric. A massless scalar field $\psi$ on this background has an Aretakis charge defined by

$$
H_{A}=\lim _{\rho \rightarrow 0} \int d^{2} x \sqrt{\gamma}\left[2 \partial_{\rho} \psi+\frac{1}{2} \frac{\partial_{\rho} \gamma}{\gamma} \psi\right],
$$

with $\gamma=\operatorname{det} \gamma_{i j}$. The Aretakis charge is conserved on the horizon (i.e., its value is $v$-independent).

Under the coordinate change

$$
\rho \rightarrow r=\frac{1}{\rho},
$$

the metric takes the form

$$
\begin{aligned}
d s^{2}= & \frac{L^{2}}{r^{2}}\left[-F d v^{2}-2 d v d r\right. \\
& \left.+r^{2} h_{i j}\left(d x^{i}-C^{i} d v\right)\left(d x^{j}-C^{j} d v\right)\right],
\end{aligned}
$$

where $C^{i}=h^{i} / r$ and $h_{i j}=\gamma_{i j} / L^{2}$. The metric inside the square brackets corresponds to a weakly asymptotically flat spacetime, in which the asymptotic 2-dimensional compact space has metric

$$
\omega_{i j}\left(x^{i}\right)=\lim _{r \rightarrow \infty} h_{i j}\left(r, x^{i}\right)=\frac{\gamma_{i j}\left(\rho=0, x^{i}\right)}{L\left(x^{i}\right)^{2}} .
$$

We call the weakly asymptotically flat metric in the square brackets of (4) the conformal dual of the initial metric with an extremal black hole.

A massless scalar field $\tilde{\psi}$ propagating in a weakly asymptotically flat spacetime has a conserved (i.e., $v$ independent) charge at future null infinity, namely the Newman-Penrose charge [10]. Its definition is:

$$
H_{\mathrm{NP}}=-\lim _{r \rightarrow \infty} \int d^{2} x \sqrt{\omega}\left[2 \partial_{r}(r \tilde{\psi})+r \partial_{r} \zeta \tilde{\psi}\right] .
$$

Here $\omega=\operatorname{det} \omega_{i j}$ and $\zeta$ is defined by the relation:

$$
h=\operatorname{det} h_{i j}=\omega \zeta^{2} .
$$

Note that weak asymptotic flatness implies $\zeta(r)=$ $1+O(1 / r)$ as $r \rightarrow \infty$.

In [9] it is shown as well that the solutions to the massless wave equation in the original spacetime and in its conformal dual are related by

$$
\psi\left(v, \rho, x^{i}\right)=\frac{L\left(x^{i}\right)}{r} \tilde{\psi}\left(v, \frac{1}{r}, x^{i}\right)
$$

It is stated in [9] that this allows us to map the Aretakis charge of the original spacetime to the NP charge of its conformal dual. However the relation is not worked out explicitly except in the particular case of the extremal Reissner-Nordström solution. In the following section we derive the general explicit relation between both charges.

\section{GENERAL RELATION BETWEEN ARETAKIS CHARGE AND DUAL NP CHARGE IN 4 DIMENSIONS}

To find the explicit general relation between $H_{A}$ and the conformal dual $H_{\mathrm{NP}}$, we write the field in the vicinity of the original spacetime's horizon in an expansion of the form:

$$
\psi=\psi_{0}+\rho \psi_{1}+O\left(\rho^{2}\right),
$$

where the $\psi_{j}$ coefficients depend on $v$ and $x^{i}$ but not $\rho$. Using this expansion in (2) leads to:

$$
H_{A}=\int d^{2} x \sqrt{\gamma}\left(2 \psi_{1}+\frac{1}{2} \frac{\gamma^{\prime}}{\gamma} \psi_{0}\right)
$$

where both $\gamma$ and its derivative are evaluated at the horizon $(\rho=0)$.

Analogously, writing the asymptotic field in the conformal dual spacetime in an expansion of the form

$$
\tilde{\psi}=\frac{\tilde{\psi}_{1}}{r}+\frac{\tilde{\psi}_{2}}{r^{2}}+O\left(1 / r^{3}\right),
$$

we get when replacing in (6) the result

$$
H_{\mathrm{NP}}=\int d^{2} x \sqrt{\omega}\left(2 \tilde{\psi}_{2}-r^{2} \zeta^{\prime} \tilde{\psi}_{1}\right)
$$

where now $\zeta^{\prime}$ stands for the $r \rightarrow \infty$ asymptotic limit of $\partial_{r} \zeta$.

From (5) and (7) we have the relation

$$
\zeta=L\left(x^{i}\right)^{-2} \frac{\sqrt{\gamma}}{\sqrt{\omega}} .
$$

From this using the chain rule, that $\rho=1 / r$, and that $\omega=\left.L^{-4} \gamma\right|_{\rho=0}$, we find:

$$
\left.\zeta^{\prime}(r)\right|_{r \rightarrow \infty}=-\left.\frac{1}{2 r^{2}} \frac{\gamma^{\prime}(\rho)}{\gamma}\right|_{\rho=0} .
$$

Therefore the NP charge reduces to

$$
H_{\mathrm{NP}}=\int d^{2} x \sqrt{\omega}\left(2 \tilde{\psi}_{2}+\left.\frac{1}{2} \frac{\gamma^{\prime}(\rho)}{\gamma}\right|_{\rho=0} \tilde{\psi}_{1}\right) .
$$

Using the relation (8) to match the expansion coefficients we see that

$$
\psi_{0}=L\left(x^{i}\right) \tilde{\psi}_{1}, \quad \psi_{1}=L\left(x^{i}\right) \tilde{\psi}_{2},
$$


which implies that

$$
H_{\mathrm{NP}}=\int d^{2} x \sqrt{\omega} L^{-1}\left(x^{i}\right)\left(2 \psi_{1}+\left.\frac{1}{2} \frac{\gamma^{\prime}}{\gamma}\right|_{\rho=0} \psi_{0}\right) .
$$

Comparing (10) with (17) we see that the "charge densities" in both expressions (i.e., the quantities that give $H_{A}$ and $H_{\mathrm{NP}}$ when integrated in two dimensions with measures $\sqrt{\gamma}$ and $\sqrt{\omega}$ respectively) are identical up to an additional $L^{-1}\left(x^{i}\right)$ factor in the second one.

Since the relation between both $2 \mathrm{~d}$ measures is found from (5), we can also summarize the relation between both charges as:

$$
\begin{gathered}
H_{A}=\left.\int d^{2} x \sqrt{\gamma}\right|_{\rho=0} h_{A}, \quad H_{\mathrm{NP}}=\left.\int d^{2} x \sqrt{\gamma}\right|_{\rho=0} h_{\mathrm{NP}} ; \\
h_{A}=L\left(x^{i}\right)^{3} h_{\mathrm{NP}} .
\end{gathered}
$$

In other words, the "charge densities" to be integrated under the same measure are related by a $L^{3}\left(x^{i}\right)$ factor.

We see that there is no straightforward mapping between the charges, in the sense that there is no operation that provides the quantity $H_{\mathrm{NP}}$ directly from the input $H_{A}$ or vice versa. Rather, it is the densities to be subjected to angular integration that are related through the factor $L\left(x^{i}\right)$ from the metric (1).

The only case in which the two charges can be directly mapped is when $L\left(x^{i}\right)$ is a constant. An example of this is the extreme Reissner-Nordström metric, where we have $L=M$ ( $M$ being the mass parameter). In this case, our result reduces immediately to the simpler relation $H_{A}=M^{3} H_{\mathrm{NP}}$. This agrees with the result of $[6,9]$.

\section{A. Example: Extremal Kerr black hole}

As a nontrivial example, in this subsection we compute the Aretakis charge and the conformal dual NP charge for the extremal Kerr black hole.

As discussed, e.g., in $[11,12],{ }^{1}$ the near-horizon regime of the extremal Kerr metric is described by Gaussian null coordinate system of the form

$$
\begin{aligned}
d s^{2}= & \left(\frac{1+x^{2}}{2}\right)\left(-\frac{\tilde{\rho}^{2}}{2 a^{2}} d v^{2}+2 d v d \tilde{\rho}\right)+a^{2}\left(\frac{1+x^{2}}{1-x^{2}}\right) d x^{2} \\
& +4 a^{2}\left(\frac{1-x^{2}}{1+x^{2}}\right)\left(d \phi+\frac{\tilde{\rho}}{2 a^{2}} d v\right)^{2},
\end{aligned}
$$

where $x=\cos \theta$.

Comparing with the form of the metric (1) on which our analysis is based, we see that the radial coordinates $\tilde{\rho}$ and $\rho$

\footnotetext{
${ }^{1}$ For higher dimensional examples of extremal near horizon geometries, see, e.g., [13].
}

are related by the rescaling $\tilde{\rho}=2 a^{2} \rho$, and that therefore we have:

$$
L^{2}(x)=a^{2}\left(1+x^{2}\right) .
$$

The angular metric functions are given by

$$
\gamma_{i j} d x^{i} d x^{j}=a^{2} \frac{1+x^{2}}{1-x^{2}} d x^{2}+4 a^{2} \frac{1-x^{2}}{1+x^{2}} d \phi^{2},
$$

whereas $h^{x}=0$ and $h^{\phi}=1$. The determinant $\gamma$ is computed from (21) to be

$$
\gamma=4 a^{4}
$$

It should be noted that all these metric functions are evaluated at the horizon. For the computation of the Aretakis charge we also need the first-order expansion of $\gamma_{i j}$ away from the horizon. This has been computed in [14]:

$$
\begin{gathered}
\gamma_{x x}^{(1)}=\frac{4 a}{1-x^{4}}, \quad \gamma_{\phi \phi}^{(1)}=\frac{16 a x^{2}\left(1-x^{2}\right)}{\left(1+x^{2}\right)^{3}}, \\
\gamma_{x \phi}^{(1)}=\frac{4 a x\left(1-x^{2}\right)}{\left(1+x^{2}\right)^{2}} .
\end{gathered}
$$

From these and the zeroth-order coefficients given in (21) it is straightforward to compute:

$$
\left.\frac{\partial_{\lambda} \gamma}{\gamma}\right|_{\lambda=0}=\frac{4}{a\left(1+x^{2}\right)}
$$

The radial expansion parameter $\lambda$ here is not the same as our radial coordinate $\rho$. It follows the form of metric used in [14]:

$$
\begin{aligned}
d s^{2}= & \lambda^{2} \bar{F}(\lambda, x) d v^{2}+2 d v d \lambda+2 \lambda \bar{h}_{i}(\lambda, x) d v d x^{i} \\
& +\gamma_{i j}(\lambda, x) d x^{i} d x^{j}
\end{aligned}
$$

This radial coordinate is related to ours by $\lambda=L^{2}(x) \rho$ in the vicinity of the horizon. Using this, (20) and (24), we can write the Aretakis charge (2) of the extremal Kerr black hole as:

$$
H_{A}=4 a^{2} \int d x d \phi\left[\left.\partial_{\rho} \psi\right|_{\rho=0}+\left.a \psi\right|_{\rho=0}\right] .
$$

Consequently, the Newman-Penrose charge of the conformal dual spacetime (written in terms of the field and its radial derivative on the original spacetime's horizon) is given by

$H_{\mathrm{NP}}=\frac{4}{a} \int d x d \phi \frac{1}{\left(1+x^{2}\right)^{3 / 2}}\left[\left.\partial_{\rho} \psi\right|_{\rho=0}+\left.a \psi\right|_{\rho=0}\right]$. 
Due to the nontrivial nature of the angular function $L(x)$, there is no direct mapping between both charges as happens in the extreme Reissner-Nordström case.

We conclude this section with some additional remarks on the relation between the original metric and its conformal dual. The extremal four-dimensional Reissner-Nordström metric has the property that its conformal dual metric, as defined above from Eq. (4), is identical to the original one (up to a constant that can be absorbed into a redefinition of the radial coordinate) [15]. Thus in the special case of extremal Reissner-Nordstrom the Aretakis charge is mapped to the NP charge of the same spacetime. A similar property is enjoyed by extremal 4-charge static black holes in ungauged four-dimensional STU supergravity, in the case where the four charges are pairwise equal [9]. These correspondences were an important part of the motivation of [9] for exploring the relation between Aretakis and NP charges. Therefore, it is interesting to explore whether extremal Kerr satisfies any similar property.

Though we do not have the full expression for the extremal Kerr metric in the horizon-adapted Gaussian null coordinate system, we have the zeroth-order metric at the horizon, which lets us know the zeroth-order asymptotic metric at $r \rightarrow \infty$ in the conformal dual spacetime. This metric is

$$
\begin{aligned}
d s^{2}= & -d v^{2}-2 d v d r+\frac{r^{2} \gamma_{i j}(x)}{L^{2}(x)}\left(d x^{i}-\frac{h^{i}(x)}{r} d v\right) \\
& \times\left(d x^{j}-\frac{h^{j}(x)}{r} d v\right),
\end{aligned}
$$

with $L$ given in (20), $\gamma_{i j}$ given in (21), and $h^{x}=0, h^{\phi}=1$ as before. It is seen that the conformal dual metric is not (asymptotically) equal to the original Kerr metric and in fact is only weakly asymptotically flat (rather than asymptotically flat) due to the nontrivial angular metric. Indeed after replacing $x=\cos \theta$ we have

$$
\frac{\gamma_{i j} d x^{i} d x^{j}}{L^{2}}=d \theta^{2}+\frac{4 \sin ^{2} \theta}{\left(1+\cos ^{2} \theta\right)^{2}} d \phi^{2}
$$

instead of $d \theta^{2}+\sin ^{\theta} d \phi^{2}$ as should be the case in an asymptotically flat spacetime. The simple relation between the extremal black hole spacetime and its conformal dual present for Reissner-Nordström spacetime is therefore not generalized to rotating black holes.

\section{DISCUSSION}

The primary goal of the present paper is to extend the results of [9] by providing an explicit expression linking the Aretakis charge of an extremal black hole spacetime with the Newman-Penrose charge of the conformal dual spacetime. By the latter we mean the conformal metric to the $\rho \rightarrow 1 / r$ inverted spacetime, as explained above [Eq. (4)]. We saw that, aside from spherically symmetric cases such as the extremal Reissner-Nordström black hole, in general there is no direct mapping between the charges. Rather, the mapping is at the level of the densities to be integrated over the horizon and over asymptotic infinity, as exhibited above through (10) and (17), or more explicitly in (18).

We have also provided an explicit computation of both charges in the extremal Kerr spacetime (and its conformal dual). This is noteworthy as a nontrivial example where the metric is not spherically symmetric. The calculation was facilitated by the previous construction in [14] of the first-order expansion for the angular near-horizon metric $\gamma_{i j}$.

The results presented in this paper should be a stepping stone towards further exploration of the near horizonasymptotic infinity duality and its consequences. One area in which additional investigations would be especially fruitful would be the extension of the results presented to higher dimensions, which was already hinted in [9] but could be made explicit along the lines of the present paper. In higher dimensions the correspondence between $H_{A}$ and $H_{\mathrm{NP}}$ can still be derived, although the dual spacetime on which $H_{\mathrm{NP}}$ is defined is less likely to admit of a physical interpretation because the definition of weak asymptotic flatness required for the derivation $(\zeta=1+O(1 / r))$ is further away from ordinary asymptotic flatness than it is in four dimensions. Exploring the issue in depth could possibly help clarify as well the physical meaning of this correspondence in four dimensions.

Another area of application which already received preliminary discussion in [9] is extremal $4 \mathrm{~d}$ black holes in STU supergravity [16-19] and their generalizations, including a study of the Aretakis charge and its dualities for the extremal version of their "subtracted geometry" limit [20-22].

\section{ACKNOWLEDGMENTS}

We would like to thank Hadi Godazgar, Mahdi Godazgar, and Christopher Pope for valuable discussions, especially on the higher dimensional generalizations of the current work. M. C. would like to thank the Mitchell Family Foundation for hospitality at the Brinsop Court workshop. The research of M. C. and A. S. is supported in part by the University of Pennsylvania Research Foundation (M. C. and A. S.), the DOE Award No. DESC0013528 (M. C.), the Fay R. and Eugene L. Langberg Endowed Chair (M.C.) and the Slovenian Research Agency (M. C.). 
[1] S. Aretakis, Stability and instability of extreme ReissnerNordstróm black hole spacetimes for linear scalar perturbations I, Commun. Math. Phys. 307, 17 (2011).

[2] S. Aretakis, Stability and instability of extreme ReissnerNordstrom black hole spacetimes for linear scalar perturbations II, Ann. Henri Poincare 12, 1491 (2011).

[3] S. Aretakis, Horizon instability of extremal black holes, Adv. Theor. Math. Phys. 19, 507 (2015).

[4] K. Murata, Instability of higher dimensional extreme black holes, Classical Quantum Gravity 30, 075002 (2013).

[5] J. Lucietti and H. S. Reall, Gravitational instability of an extreme Kerr black hole, Phys. Rev. D 86, 104030 (2012).

[6] J. Lucietti, K. Murata, H. S. Reall, and N. Tanahashi, On the horizon instability of an extreme Reissner-Nordstróm black hole, J. High Energy Phys. 03 (2013) 035.

[7] P. Bizon and H. Friedrich, A remark about wave equations on the extreme Reissner-Nordstróm black hole exterior, Classical Quantum Gravity 30, 065001 (2013).

[8] S. Bhattacharjee, B. Chakrabarty, D. D. K. Chow, P. Paul, and A. Virmani, On late time tails in an extreme ReissnerNordström black hole: frequency domain analysis, Classical Quantum Gravity 35, 205002 (2018).

[9] H. Godazgar, M. Godazgar, and C. N. Pope, Aretakis charges and asymptotic null infinity, Phys. Rev. D 96, 084055 (2017).

[10] E. T. Newman and R. Penrose, New conservation laws for zero rest-mass fields in asymptotically flat space-time, Proc. R. Soc. A 305, 175 (1968).

[11] H. K. Kunduri, J. Lucietti, and H. S. Reall, Near-horizon symmetries of extremal black holes, Classical Quantum Gravity 24, 4169 (2007).
[12] H. K. Kunduri and J. Lucietti, A classification of nearhorizon geometries of extremal vacuum black holes, J. Math. Phys. (N.Y.) 50, 082502 (2009).

[13] D. D. K. Chow, M. Cvetič, H. Lü, and C. N. Pope, Extremal black hole/CFT correspondence in (gauged) supergravities, Phys. Rev. D 79, 084018 (2009).

[14] C. Li and J. Lucietti, Transverse deformations of extreme horizons, Classical Quantum Gravity 33, 075015 (2016).

[15] W.E. Couch and R. J. Torrence, Conformal invariance under spatial inversion of extreme Reissner-Nordström black holes, Gen. Relativ. Gravit. 16, 789 (1984).

[16] M. Cvetič and D. Youm, Dyonic BPS saturated black holes of heterotic string on a six torus, Phys. Rev. D 53, R584 (1996).

[17] M. Cvetič and A. A. Tseytlin, Solitonic strings and BPS saturated dyonic black holes, Phys. Rev. D 53, 5619 (1996); Erratum, Phys. Rev. D55, 3907(E) (1997).

[18] M. Cvetič and D. Youm, Entropy of nonextreme charged rotating black holes in string theory, Phys. Rev. D 54, 2612 (1996).

[19] D. D. K. Chow and G. Compère, Black holes in $N=8$ supergravity from $\mathrm{SO}(4,4)$ hidden symmetries, Phys. Rev. D 90, 025029 (2014).

[20] M. Cvetič and F. Larsen, Conformal symmetry for general black holes, J. High Energy Phys. 02 (2012) 122.

[21] M. Cvetič and F. Larsen, Conformal symmetry for black holes in four dimensions, J. High Energy Phys. 09 (2012) 076.

[22] M. Cvetič and F. Larsen, Black holes with intrinsic spin, J. High Energy Phys. 11 (2014) 033. 